\title{
Acquisition and processing pitfall associated with clipping near-surface seismic reflection traces
}

\author{
Steven D. Sloan ${ }^{1}$, Don W. Steeples ${ }^{1}$, and Peter E. Malin ${ }^{2}$
}

\begin{abstract}
The processing of clipped seismic traces may produce high-frequency wavelets that can be misinterpreted as reflections in filtered shot gathers and common-midpoint (CMP) stacked sections. To illustrate this effect, a near-surface CMP seismic reflection survey was conducted using two sources to compare the effects of various band-pass frequency filters on clipped traces. An event observed in the clipped data set replicated the frequency of the filter operators applied, similar to the effect of convolving a boxcar function with the filter operator. The anomaly exhibited hyperbolic moveout and imitated a reflection during the processing stages. The hyperbolic event was flattened by NMO corrections chosen for the target reflection, and it stacked in as a coherent event in the final section. Clipped data should be removed or corrected before processing to prevent misinterpreting high-frequency reflection artifacts in trace gathers and stacked sections.
\end{abstract}

\section{INTRODUCTION}

Shallow seismic reflection data are commonly collected by using dense receiver and source spacings, which are required to image shallow reflectors and to prevent spatial aliasing of data. The close proximity of the source and the nearest receivers resulting from the short offsets can result in clipped traces in which peaks and/or troughs have been squared off, depending on the energy of the source selected. Ultrashallow surveys might require a receiver spacing of $20 \mathrm{~cm}$ or less, which can result in a relatively high percentage of clipped traces in the data set (Figure 1). This article addresses a potential pitfall associated with data acquisition and processing in which clipped surface waves can be misinterpreted as high-frequency reflections on filtered shot gathers and on stacked sections.

When clipped seismic traces are processed digitally, high-frequency artifacts can appear as reflections in field records and stacked sections. Galbraith and MacMinn (1982) observe the effects of deconvolution on clipped traces, noting that each clipped peak or trough is split into two or more individual peaks and troughs after deconvolution. This leads to the presence of anomalous peaks in processed stacked sections that could lead to their being misinterpreted as a stratigraphic feature or other anomaly. The presence of anomalous peaks is not a problem if clipped traces are corrected or removed from the data set. Possible solutions for eliminating gain errors such as clipping include muting, filtering, polynomial interpolation (Galbraith and MacMinn, 1982), minimum error energy operators (MEEOs) (Nyman, 1977; Galbraith and MacMinn, 1982), and adaptive lattice filters (Khan, 1982).

Muting can eliminate the clipped portion of traces but also can be tedious and time-consuming, especially for large data sets. Filtering can smooth gain-associated errors if they are not too severe; however, this method can produce additional problems for clipped traces. Nyman (1977) discusses the use of MEEOs for error correction. Galbraith and MacMinn (1982) determine that the MEEO method is more effective at correcting gain-associated errors and clipping than polynomial interpolation is. Yet another method, described by Khan (1982), uses adaptive lattice filters to correct errors.

Although our article points out methods for correcting clipping errors, the primary focus is to show problems that can propagate through the data-processing stages and negatively impact data interpretation if clipped traces are not recognized and corrected or removed.

\section{DATA ACQUISITION AND PROCESSING}

To illustrate how high-pass filtering of clipped data can produce artifacts on stacked common-midpoint (CMP) data, we conducted two CMP seismic reflection surveys using identical acquisition parameters, with the exception of the source. The receiver line consisted of 144 Mark Products L40-A 100-Hz vertical geophones planted with a 10 -cm interval. Data were recorded using a 0.125 -ms sampling interval for 128 ms by two 72-channel Geometrics StrataView seismographs with 24-bit analog-to-digital (A/D) conversion.

\footnotetext{
Manuscript received by the Editor 2 May 2007; revised manuscript received 25 July 2007; published online 29 November 2007.

${ }^{1}$ The University of Kansas, Department of Geology, Lawrence, Kansas, U.S.A. E-mail: sloansd@ku.edu; don@ku.edu.

${ }^{2}$ University of Auckland, Institute of Earth Science and Engineering, Auckland, New Zealand. E-mail: p.malin@ auckland.ac.nz. (C) 2008 Society of Exploration Geophysicists. All rights reserved.
} 
The first survey was collected using a .22-caliber rifle firing longrifle ammunition and a shot interval of $20 \mathrm{~cm}$. The second survey was conducted in the same manner using a .223 -caliber rifle with a 55 -grain bullet as the source. Data processing for the two data sets was identical and included geometry definition, CMP sorting, NMO

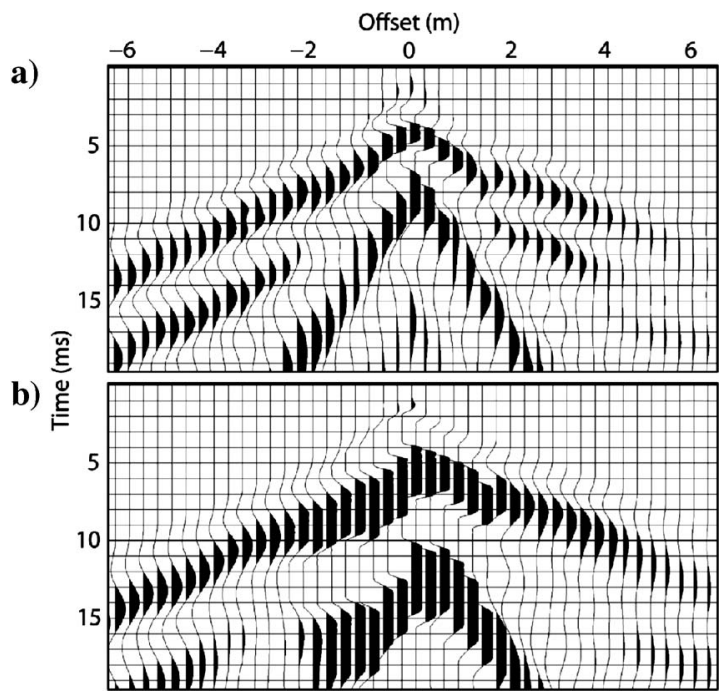

Figure 1. Field files acquired at the same source location using (a) a .22 -caliber and (b) a .223-caliber rifle. (a) Clipped traces are out to $0.6 \mathrm{~m}$ from the source. (b) The clipping reaches as far as $2.0 \mathrm{~m}$. The data are presented without any filtering, although a $9-\mathrm{dB}$ attenuation was applied to prevent trace overlap.

a)

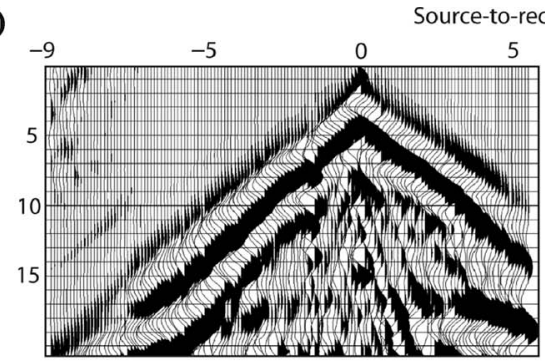

$5 \quad-9$

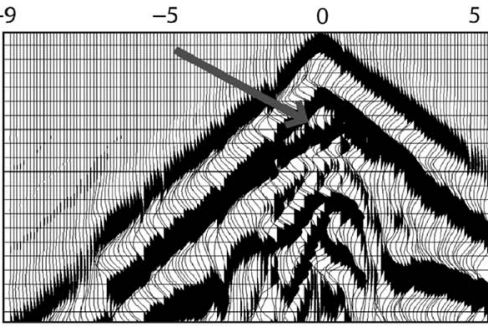

b)
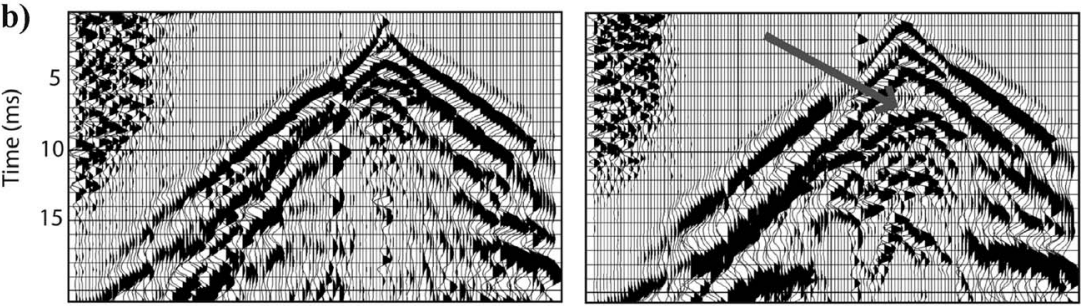

c)
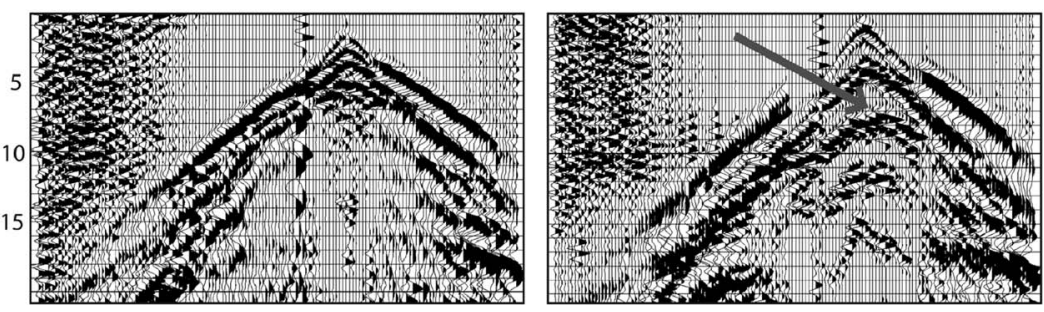

Figure 2. Field records collected with the .22-caliber rifle (left) and .223-caliber rifle (right) at the same location. Data are displayed with band-pass filters of (a) 200-500, (b) 600-900, and (c) 1000-1300 Hz. corrections, CMP stacking, band-pass filtering, and automatic gain control (AGC) to produce a brute stack. Early muting was not applied, which allowed the clipped-trace effects to be seen on the entire shot records.

\section{RESULTS}

Figure 2 shows shot records from a single source location collected with the .22- and .223-caliber rifles with various band-pass filters applied. The target reflection from the water table is located at approximately $16 \mathrm{~ms}$. Although the records are similar, the second arrival in the data collected with the .223-caliber rifle exhibits a separation at approximately $6 \mathrm{~ms}$ (indicated by the arrow, Figure 2) that is not seen in the data collected by the .22 -caliber rifle. This separation becomes more pronounced as the frequency of the applied filter increases.

This event, which also appears to have a hyperbolic moveout, increases in frequency as the filter's frequency increases, without becoming distorted or decreasing in amplitude. The hyperbolic appearance could be mistaken for a very shallow reflection and, if left in the data set, might stack coherently. Hyperbola fitting of this pseudoreflection yields a velocity of approximately $300 \mathrm{~m} / \mathrm{s}$, slower than the velocities of both the water-table reflection $(450-550 \mathrm{~m} / \mathrm{s})$ and the direct wave $(400 \mathrm{~m} / \mathrm{s})$.

Velocity picks obtained from the water-table reflection at every fifth CMP location, ranging from 450 to $550 \mathrm{~m} / \mathrm{s}$, were used to apply NMO corrections to both data sets. Figure 3 shows the effects of the NMO corrections on the pseudoreflection in CMP gathers from the .22-caliber and .223-caliber data sets at a coincident location. The pseudoreflection event at about $7 \mathrm{~ms}$ has been flattened and is coherent in the .223 data but not in the CMP gather of the .22 data.

Figure 4 shows the brute stacks of both the .22 and .223 data with clipped traces included (Figure 4a), clipped portions surgically muted (Figure $4 \mathrm{~b}$ ), and clipped traces removed entirely (Figure 4c). The data are displayed with a $600-900-\mathrm{Hz}$ band-pass filter with 18-dB/octave roll-off slopes and no mutes. A coherent event at approximately $7 \mathrm{~ms}$ in the .223 stack, indicated by the arrow in Figure $4 \mathrm{a}$, is not present in the .22 stack. This event corresponds to the event observed in the field records and NMO-corrected CMP gathers. Clipped traces in the .22 data ranged from $\pm 0.6 \mathrm{~m}$ source-to-receiver offset represented by 13 traces, or $9 \%$ of the 144 . The .223 data overpowered the geophones as far as $2.0 \mathrm{~m}$ from the source, representing 41 of the 144 traces $(28 \%)$ collected at each source location (Figure 1).

\section{DISCUSSION}

Figure 5 illustrates the effects of various bandpass filters with increasing frequency on an unclipped and clipped seismic trace and the corresponding amplitude spectra. As filters with higher band-pass frequency are applied to the unclipped trace, high-frequency ring is introduced. The 
traces are recognizably noisier, and the signal is diminished because of the decreasing bandwidth. However, when the same filters are applied to a clipped trace, the filter operator is replicated at the squared wavelet corners where the clipping occurs.

The largest peaks and troughs in each of the filtered clipped traces occur at the same position as the squared corners where clipping occurs in the original trace, as indicated by the shaded area. This is essentially equivalent to convolving the filter operator with a boxcar function. Ringing does not occur in the filtered clipped traces when the frequency of the filter operator is increased, because the band- width increases artificially as additional cycles are introduced where clipping occurs.

Corresponding amplitude spectra are shown for traces a, c, g, k, and $\mathrm{o}$. As the frequency of the filter is increased, the spectra of the unclipped traces also become increasingly noisier. Those of the clipped traces retain the same shape and smoothness across the frequency axis. Table 1 shows the passband of the filters applied to each trace.

Data demonstrate the effects of some of the processing steps that may prevent this problem by comparing brute stacks, including the clipped traces (Figure 4a), the clipped data surgically muted (Figure
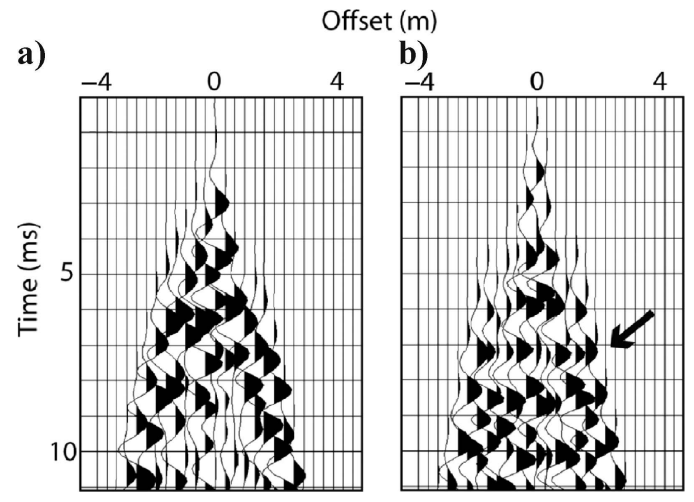

Figure 3. Coincident NMO-corrected CMP gathers from the (a) .22-caliber and (b) .223 -caliber data sets. The event near $7 \mathrm{~ms}$, indicated by the arrow, has become flattened and coherent after NMO corrections were applied with a $15 \%$ stretch mute, using velocity picks from the targeted water-table reflection. Data are displayed with a 600-900-Hz band-pass filter without mutes or gains applied.

a)

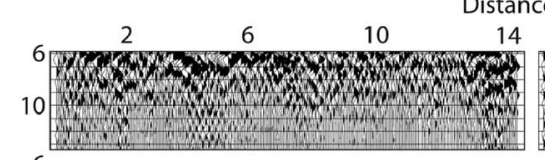

Distance (m)

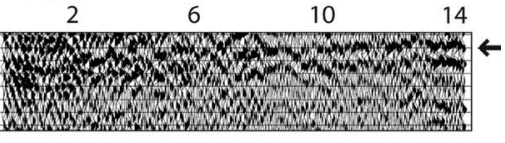

b)

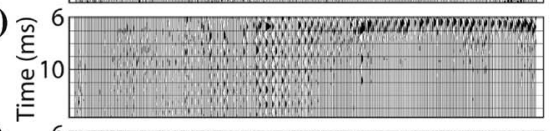

c)
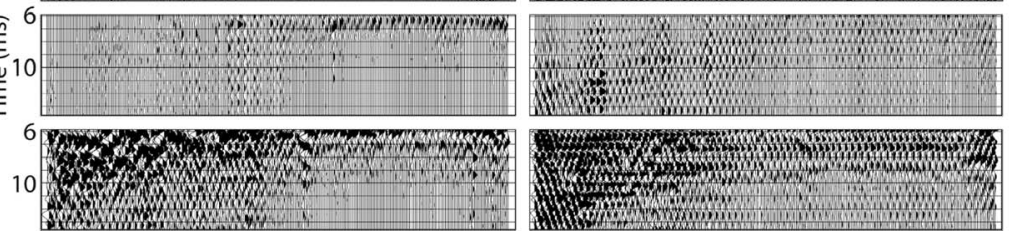

Figure 4. Brute stacks of the .22-caliber (left) and .223-caliber (right) data. Both data sets have been processed identically for comparison purposes. (a) A coherent event near $7 \mathrm{~ms}$ in the .223 data, indicated by the arrow, is not evident in the .22 data. (b) A surgical mute applied to remove the clipped portion of the traces. (c) The brute stacks after the clipped traces have been removed entirely. a)

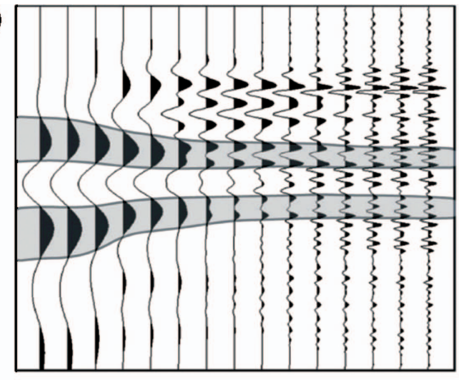

b)

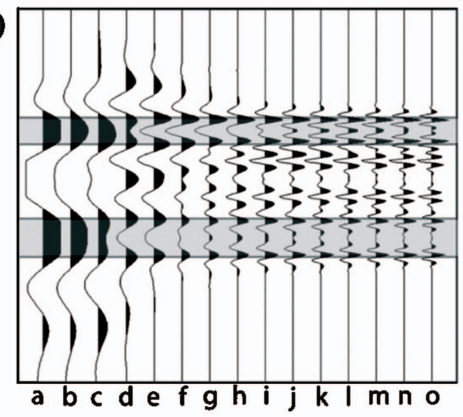

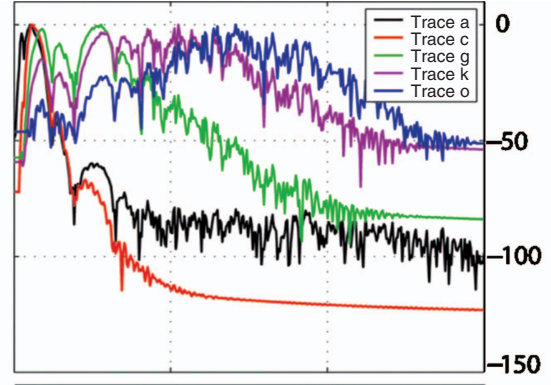

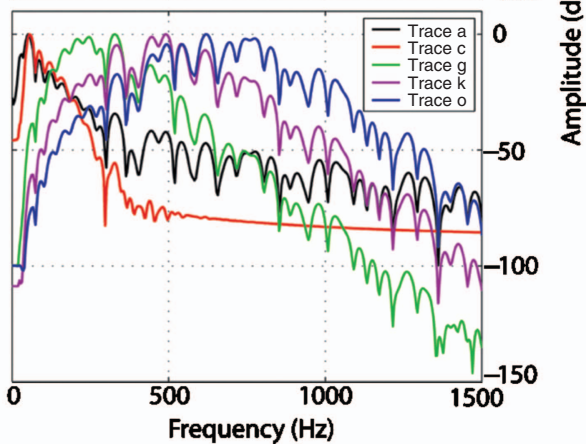

Figure 5. (a) An unclipped and (b) a clipped trace with various band-pass filters applied, increasing in frequency (traces a through o) from left to right. The corresponding amplitude spectra are shown on the right. View (a) begins to ring at higher frequencies. In (b), the wavelet does not show the same ring but appears to increase in frequency as higher-frequency band-pass filters are applied to it. The highfrequency wavelet seen in (b) is the filter operator convolved with the corners at which clipping occurs in the data. Filter specifications for each trace are described in Table 1. 
4b), and the clipped traces removed (Figure 4c). As seen in Figure $4 \mathrm{a}$, processing the clipped traces can result in artifacts that appear as high-frequency reflections, which can lead to misinterpretations. Surgically muting the clipped portion of the traces, as in Figure $4 \mathrm{~b}$,

Table 1. Band-pass filters (Hertz) applied to each trace in Figure 5. Filters were applied with 18-dB/octave roll-off slopes.

\begin{tabular}{cc}
\hline Trace & Filter passband $(\mathrm{Hz})$ \\
\hline a & N/A \\
b & $0-300$ \\
c & $100-400$ \\
d & $200-500$ \\
e & $300-600$ \\
f & $400-700$ \\
g & $500-800$ \\
h & $600-900$ \\
i & $700-1000$ \\
j & $800-1100$ \\
k & $900-1200$ \\
l & $1000-1300$ \\
m & $1100-1400$ \\
n & $1200-1500$ \\
o & $1300-1600$ \\
\hline
\end{tabular}

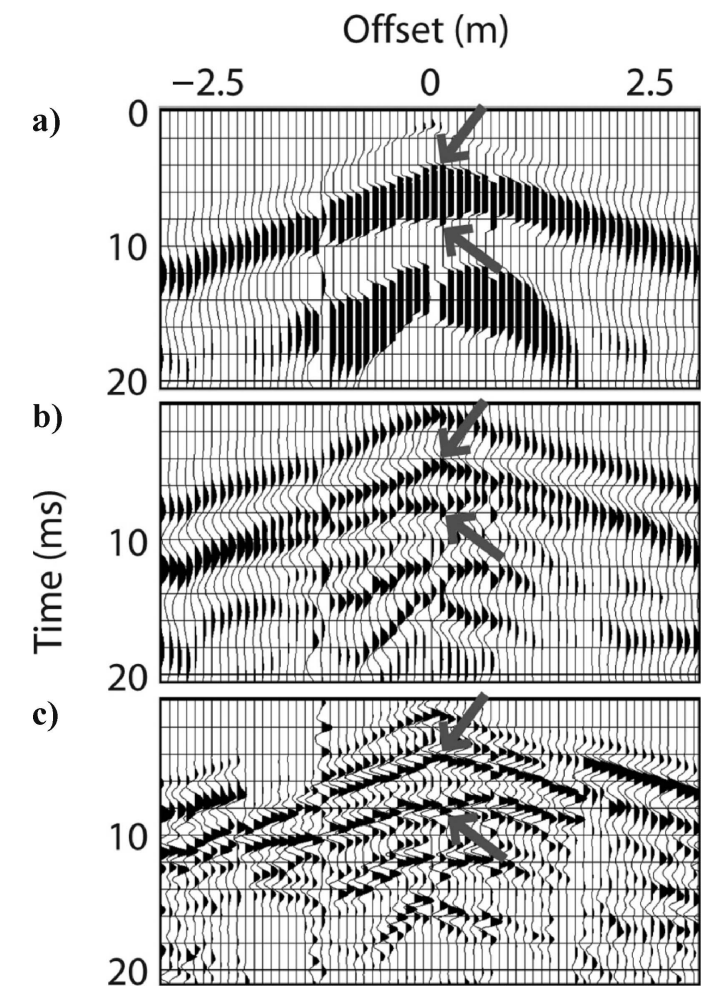

Figure 6. The same shot gather is shown with (a) no filtering, (b) a 200-500-Hz band-pass filter, and (c) a 600-900-Hz band-pass filter. Arrows point to the same event as it changes with each filter. Filters were applied with 18-dB/octave roll-off slopes. The top shot gather has a $9-\mathrm{dB}$ attenuation applied to prevent trace overlap. effectively eliminates the artifact; however, this method may be time-consuming and tedious. The most reliable way is to omit the clipped traces from the data set, as illustrated in Figure 4c.

Omitting the clipped traces may be fast and reliable. However, in the case with the .223-caliber rifle, that would reduce the total number of traces by about $30 \%$ and would lower the fold significantly. In that case, an early mute designed to delete clipped data might be the most efficient method for removal because an early mute is necessary to remove the direct and refracted waves and would be applied to the data anyway. If removing the traces or muting is not a viable option, the minimum-error energy operator reported by Nyman (1977) and discussed by Galbraith and MacMinn (1982) could be used to attempt to correct clipping errors.

The hyperbolic appearance of the reflection event is from a combination of clipping and frequency filtering. Figure 6 illustrates the same shot gather with no filter applied (Figure 6a), a 200-500-Hz band-pass filter (Figure 6b), and a 600-900-Hz band-pass filter (Figure $6 \mathrm{c}$ ). The traces in the shot gather behave in the same manner as those described in Figure 5.

The event indicated by the arrows in the raw shot gather is linear. However, as frequency filters are applied, the squared corners become individual peaks and troughs. That creates the separation observed in the same event in the shot gathers shown in Figure $6 \mathrm{~b}$ and $\mathrm{c}$, where the top of the event remains linear and the bottom appears hyperbolic. The separation appears to enlarge with increasing frequency of the filters, but the separated events are actually at the same times in each gather, as indicated by arrows. As amplitudes decrease with offset and clipping diminishes, the clipped corners and filter-induced peaks come closer together, giving the event its hyperbolic appearance.

\section{CONCLUSION}

The processing of clipped seismic traces has the potential of creating artifacts that could be misinterpreted as high-frequency reflections in shot gathers and stacked sections. As filters of increasingly higher band-pass frequency are applied to an unclipped trace, the high-frequency noise level increases because of the decreased bandwidth. In contrast, the frequency of a clipped trace mirrors that of the filter applied to it by replicating the filter operator. It will continue to increase in frequency without distortion or without adding high-frequency noise to the wavelet, and it exhibits no change in the bandwidth.

Clipped data should be removed by muting or trace editing or they should be corrected during the preprocessing stage to avoid the problems described. Field testing of potential seismic sources prior to conducting a survey is recommended and can prevent a problem by selecting the source with the appropriate energy to meet the survey objectives. Clipping can occur as a result of physical limitations of geophones or by setting all the bits that can be recorded by a digital sample to ones. It might be beneficial to check the data for digital samples which are the largest possible number that can come from the A/D converter, possibly indicating the presence of clipped traces.

As with all seismic projects, care should be taken during the processing stages to ensure that quality control is maintained and that 
the processing steps or selected parameters do not introduce unwanted artifacts into the data set.

\section{ACKNOWLEDGMENTS}

The authors would like to thank Paul Vincent, Robert Eslick, Gerard Czarnecki, and George Tsoflias for their time in the field; Sally Hayden for editorial assistance; and the editors and reviewers for their constructive comments. This research was supported by the Office of Science (BER), U. S. Department of Energy, grant DE-FG0203ER63656. However, any opinions, conclusions, or recommenda- tions expressed herein are those of the authors and do not necessarily reflect the views of the DOE.

\section{REFERENCES}

Galbraith, M., and C. L. MacMinn, 1982, Error correction in field recorded seismic data: Journal of the Canadian Society of Exploration Geophysicists, 18, 23-33.

Khan, R. H., 1982, Seismic data error correction using adaptive lattice filters: 52nd Annual International Meeting, SEG, Expanded Abstracts, 124-126.

Nyman, D., 1977, The interpolation error operator, Time series error detection and correction: Geophysics, 42, 773-777. 